\title{
Conservation of the "Outside-in" Germination Pathway in Paraclostridium bifermentans
}

\author{
Disha Bhattacharjee and Joseph A. Sorg* \\ Department of Biology, Texas A\&M University, College Station, TX, United States
}

Clostridium difficile spore germination is initiated in response to certain bile acids and amino acids (e.g., glycine). Though the amino acid-recognizing germinant receptor is unknown, the bile acid germinant receptor is the germination-specific, subtilisin-like pseudoprotease, CspC. In C. difficile the CspB, CspA, and CspC proteins are involved in spore germination. Of these, only CspB is predicted to have catalytic activity because the residues important for catalysis are mutated in the $\operatorname{csp} A$ and $\operatorname{csp} C$ sequence. The CspB, CspA, and CspC proteins are likely localized to the outer layers of the spore (e.g., the cortex or the coat layers) and not the inner membrane where the Ger-type germinant receptors are located. In C. difficile, germination proceeds in an "outside-in" direction,

OPEN ACCESS

Edited by: Biswarup Mukhopadhyay, Virginia Tech, United States

Reviewed by: Aimee Shen,

Tufts University School of Medicine, United States

Stanley Brul,

University of Amsterdam, Netherlands

*Correspondence:

Joseph A. Sorg

jsorg@bio.tamu.edu

Specialty section:

This article was submitted to Microbial Physiology and Metabolism,

a section of the journal

Frontiers in Microbiology

Received: 17 August 2018 Accepted: 28 September 2018 Published: 17 October 2018

Citation:

Bhattacharjee $D$ and Sorg JA

(2018) Conservation of the "Outside-in" Germination Pathway in Paraclostridium bifermentans. Front. Microbiol. 9:2487. doi: 10.3389/fmicb.2018.02487 instead of the "inside-out" direction observed during the germination of Bacillus subtilis spores. During $C$. difficile spore germination, cortex fragments are released prior to the release of 2,4-dipicolinic acid (DPA) from the spore core. This is opposite to what occurs during $B$. subtilis spore germination. To understand if the mechanism $C$. difficile spore germination is unique or if spores from other organisms germinate in a similar fashion, we analyzed the germination of Paraclostridium bifermentans spores. We find that $P$. bifermentans spores release cortex fragments prior to DPA during germination and the DPA release from the $P$. bifermentans spore core can be blocked by high concentrations of osmolytes. Moreover, we find that $P$. bifermentans spores do not respond to steroidlike compounds (unlike the related $C$. difficile and $P$. sordellii organisms), indicating that the mere presence of the Csp proteins does permit germination in response to steroid compounds. Our findings indicate that the "outside in" mechanism of spore germination observed in $C$. difficile can be found in other bacteria suggesting that this mechanism is a novel pathway for endospore germination.

Keywords: spore, germination, Clostridium, DPA, cortex

\section{INTRODUCTION}

The endospore-forming Paraclostridium bifermentans belongs to the Clostridia family and P. bifermentans subsp. malaysia is the only known anaerobic larvicidal toxin producer whose toxins target Anopheles and Aedes mosquitoes (Qureshi et al., 2014). Due to the anaerobic nature of $P$. bifermentans vegetative cells, the organism likely survives between hosts in the form of a dormant spore. Endospore formation is conserved in many Bacilli and Clostridia, though 
the sporulation pathway exhibits some differences between organisms (Fimlaid et al., 2013). Despite these differences, the overall architecture of the metabolically dormant spore is conserved.

Located in the center of the spore, the core contains DNA, RNA, ribosomes, and protein, and the core has a low water content with high amounts of 2,4-dipicolinic acid (DPA), which provides resistance against heat (Permpoonpattana et al., 2011; Setlow, 2014). Surrounding the spore core is a thin germ cell wall, which becomes the cell wall peptidoglycan of the vegetative cell upon germination (Setlow, 2014), and a thick cortex peptidoglycan layer composed of $\mathrm{N}$-acetylglucosamine (NAG), N-acetylmuramic acid (NAM), and muramic- $\delta$-lactam residues. Finally, layers of coat proteins surround the cortex layer and protect the spore from environmental insults.

During spore development, receptors that identify suitable environmental conditions for metabolism and growth are incorporated into the spores by the mother cell or the forespore (Errington, 2003; Francis et al., 2013; Bhattacharjee et al., 2016b). Upon binding to small molecule germinants, these receptors trigger the irreversible germination process (Bhattacharjee et al., 2016b). The germination process has been described best in the model spore-forming bacterium, Bacillus subtilis. B. subtilis spores germinate in response to L-alanine or a mixture of L-asparagine, glucose, fructose and potassium ions (AGFK) (Setlow, 2014). These germinants are thought to interact with their respective germinant receptors embedded within the inner spore membrane (Moir et al., 2002; Setlow, 2014). Whereas the GerBA-BB-BC and GerKA-KB-KC germinant receptor responds to $\mathrm{AGFK}$, the GerAA-AB-AC germinant receptor responds to L-alanine (Setlow, 2014). Though the signals that activate spore germination in other organisms vary, nearly all endosporeforming organisms, studied to date, encode orthologs of the transmembrane Ger-type germinant receptor (Paredes-Sabja et al., 2011). Germinant receptor activation leads to the release of monovalent cations and the large depot of DPA, from the channel composed of the SpoVA proteins, resulting in rehydration of the spore core (Setlow, 2014). Subsequently, two redundant spore cortex lytic enzymes (SCLEs), CwlJ and SleB, are activated and, through their combined actions, the cortex is degraded (Setlow, 2014). Cortex degradation allows for full core rehydration, loss of dormancy, restoration of metabolism and, finally, outgrowth of a vegetative cell from the germinated spore.

Unlike what is found in B. subtilis and other spore-forming bacteria, Clostridium difficile [also Clostridioides difficile (Lawson et al., 2016; Oren and Garrity, 2016)] does not encode orthologs of the known ger-type germinant receptors, suggesting that C. difficile spore germination occurs through a novel mechanism or uses novel signals (Sebaihia et al., 2006). C. difficile initiates germination in response to cholic acid derivatives (bile acids) and amino acids (e.g., glycine or alanine), while chenodeoxycholic acid derivatives are competitive inhibitors of cholic acidmediated germination (Sorg and Sonenshein, 2008, 2009, 2010; Paredes-Sabja et al., 2014; Bhattacharjee et al., 2016b). Though necessary for $C$. difficile spore germination, bile acids are not sufficient (Sorg and Sonenshein, 2008). A second, amino acidbased signal is required to activate the germination process.
Glycine is the best co-germinant, but most other amino acids can substitutes with varying efficiencies (Sorg and Sonenshein, 2008; Shrestha et al., 2017; Shrestha and Sorg, 2018). Recently, calcium was reporting to function as an enhancer of $C$. difficile spore germination, however, it is unclear if it acting in the role of a bona fide germinant or if it functions as a cofactor/essential component of germination proteins (Kochan et al., 2017). Previously, our lab determined that the bile acid germinant receptor is the germination-specific, subtilisin-like, pseudoprotease, CspC (Francis et al., 2013; Paredes-Sabja et al., 2014; Bhattacharjee et al., 2016b). The Csp proteases were originally studied in Clostridium perfringens (Shimamoto et al., 2001; Masayama et al., 2006; Paredes-Sabja et al., 2011). In C. perfringens, the CspA, CspB, and CspC proteases cleave the inactive SCLE, pro-SleC, to its active form resulting in cortex degradation (Shimamoto et al., 2001). The C. perfringens CspB, CspA, and CspC proteins can be extracted from spore coats (Shimamoto et al., 2001). But, in the article that describes this, no control was given for cortex-localized proteins suggesting that these proteins could be coat- or cortex-localized (Shimamoto et al., 2001). In $C$. difficile, the $\operatorname{csp} B$ and $\operatorname{csp} A$ sequences are fused, translationally, and $\operatorname{csp} C$ is encoded downstream of $\operatorname{csp} B A$ (Adams et al., 2013). Though CspB is produced as a fusion with CspA, the timing for interdomain cleavage and the fate of CspA after cleavage is still unknown. However, the loss of CspA leads to a significant decrease in spore germination, and CspA has been shown to control CspC levels in the spore (Francis et al., 2013; Kevorkian et al., 2016). CspB is capable of processing pro-SleC to its active form. However, the catalytic triads that are characteristic of subtilisin-like proteases are absent in both CspA and CspC, and loss of $\operatorname{csp} A$ or $\operatorname{csp} C$ negatively affects spore germination, suggesting that $\mathrm{CspA}$ and $\mathrm{CspC}$ function in a regulatory role and not a catalytic role (Adams et al., 2013; Francis et al., 2013).

Based upon the predicted location of CspB, CspA, CspC, and SleC (near the cortex layer and not in/on the inner spore membrane) (Miyata et al., 1997), we hypothesized that C. difficile spore germination may be initiated differently than what had been described in other endospore-forming bacteria (Francis et al., 2015). Specifically, we hypothesized that bile acids and glycine would stimulate cortex degradation prior to the release of DPA from the spore core (a process opposite to what is observed during B. subtilis spore germination). Indeed, cortex degradation precedes release of DPA from the spore core and the release of DPA is dependent on the osmotic changes that occur at the inner membrane when cortex is degraded (Francis et al., 2015; Francis and Sorg, 2016). This suggests that C. difficile spore germination proceeds through a novel spore germination pathway where the germinants stimulate cortex degradation. In order to understand if this mechanism of germination is unique to $C$. difficile or if other organisms share this pathway of spore germination, we analyzed germination in $P$. bifermentans. $P$. bifermentans encodes a csp locus that is similar to what is observed in $C$. difficile where the $\operatorname{csp} B$ and $\operatorname{csp} A$ sequences are translationally fused and $\operatorname{csp} A$ and $\operatorname{csp} C$ do not encode proteins with complete catalytic triads. Moreover, $P$. bifermentans encodes a peptidoglycan binding protein that is $56 \%$ similar to the peptidoglycan degrading protein SleC from C. difficile. Herein, we 
find that $P$. bifermentans cortex degradation precedes the release of DPA from the spore core and the release of DPA can be delayed by high concentrations of osmolytes. Our data suggest that, like C. difficile, P. bifermentans spores germinate through an "outsidein" mechanism and add to the list of organisms that germinate through this novel pathway of spore germination.

\section{RESULTS}

\section{Identifying Potential P. bifermentans Germination Receptors}

Prior research from our laboratory demonstrated that there are differences between mechanisms for the initiation of spore germination observed in B. subtilis and C. difficile (Francis et al., 2015; Francis and Sorg, 2016). The primary difference is the presence of csp-type germinant receptors and the absence of gertype receptors in C. difficile (Paredes-Sabja et al., 2011; Francis et al., 2013). P. bifermentans also encodes homologs to C. difficile $\operatorname{csp} B A$ and $\operatorname{csp} C$ (Figure 1A). In the unannotated P. bifermentans whole genome sequence, the $\operatorname{csp} B A$ gene encodes a truncated protein. Upon sequencing the $P$. bifermentans cspBA gene, we found that there is a sequencing error in the $\operatorname{csp} B A$ gene in the deposited NCBI sequence in the form of a deletion of an adenine within a stretch of consecutive adenine residues (Supplementary Figure S1). This indicates that the P. bifermentans CspBA protein sequence is intact and has the potential to function similarly to what is observed in C. difficile.

To determine if $P$. bifermentans encodes a gerAA ortholog, we used BLAST to search the $P$. bifermentans genome for the B. subtilis GerAA protein. Interestingly there is an annotated gerA sequence in P. bifermentans. Using this protein sequence as a query, we found that the P. bifermentans sequence mostclosely matches the B. subtilis SpoVAF spore protein and not GerA. Taken together, these results suggest that $P$. bifermentans does not encode ger-type germinant receptors but encodes a csp locus that is similar to that of $C$. difficile.

\section{Germination of $P$. bifermentans Spores in Response to Amino Acids}

Previously, the germinants for $P$. bifermentans spores were identified (Gibbs, 1964; Waites and Wyatt, 1971). In order to dissect the mechanism of $P$. bifermentans spore germination, we monitored germination using both change in $\mathrm{OD}_{600} \mathrm{~nm}$ (which measures the sum of events during loss of dormancy) and release of DPA (as measured by $\mathrm{Tb}^{3+}$ fluorescence). Purified spores were suspended in HEPES-buffer alone or supplemented with L-alanine (A), L-arginine (R), L-phenylalanine (F), or all three amino acids (ARF). P. bifermentans spores rapidly germinated in the presence of ARF but not when exposed to the amino acids individually (Figure 1B). When DPA release was assayed, ARF stimulated the rapid release of DPA from the germinating $P$. bifermentans spores (Figure 1C). Next, we tested whether binary combinations of the amino acids could stimulate $P$. bifermentans spore germination. Though $\mathrm{ARF}$ was the best activator of spore germination, $\mathrm{AF}$ and

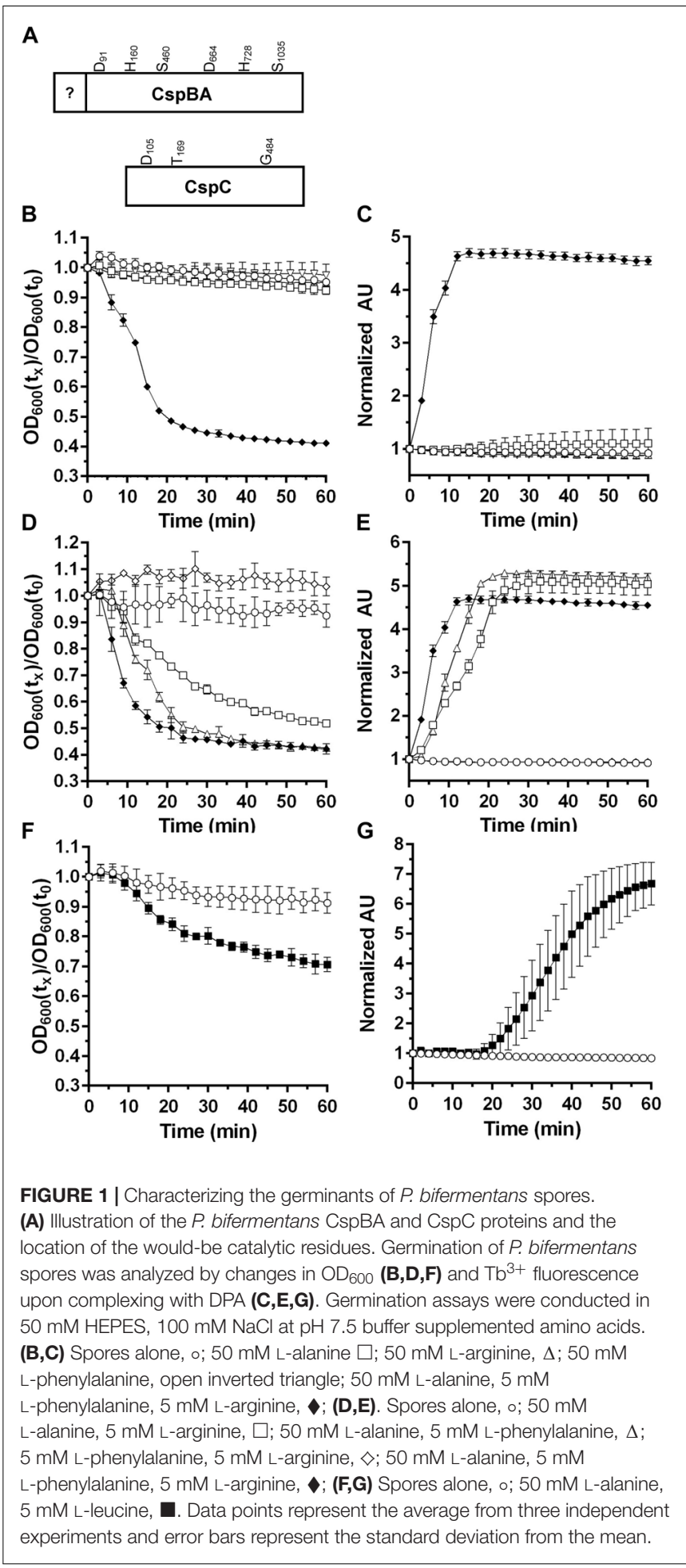

AR could also stimulate germination as measured by OD change (Figure 1D) and DPA release (Figure 1E). We then tested other amino acids to understand if other amino acids can synergize with L-alanine to stimulate $P$. bifermentans spore germination. In doing so, we found that L-leucine can function as a germinant with L-alanine as measured by both 
germination at $\mathrm{OD}_{600}$ (Figure 1F) and DPA release (Figure 1G). As determined by the rate of $\mathrm{OD}$ change and the rate of DPA release, AF was a weaker activator of spore germination than ARF, AR was weaker than $\mathrm{AF}$ and $\mathrm{AL}$ was weaker than $\mathrm{AR}(\mathrm{ARF}>\mathrm{AF}>\mathrm{AR}>\mathrm{AL})$. These results suggest that $P$. bifermentans spore germination requires at least $\mathrm{L}$-alanine and another amino acid (L-arginine, L-phenylalanine, or L-leucine) and that L-alanine is an essential, but not sufficient, germinant for P. bifermentans spores.

\section{Bile Acids Do Not Influence P. bifermentans Spore Germination}

Clostridium difficile germination is activated in response to a combination of cholic acid-class bile acids and an amino acid (e.g., glycine) (Sorg and Sonenshein, 2008; Howerton et al., 2011; Bhattacharjee et al., 2016b). In Clostridium sordellii, a related organism, ARF-mediated spore germination is enhanced by steroid-like compounds, including bile acids, and $C$. sordellii encodes orthologs of $C$. difficile $\operatorname{csp} B A$ and $\operatorname{csp} C$ (Liggins et al., 2011). Therefore, we hypothesize that steroid/bile acid recognition may be a property of organisms that encode the cspBAC locus. To determine whether bile acids can be recognized in $P$. bifermentans, we germinated $P$. bifermentans spores in presence of various bile acids [taurocholic acid (TA), deoxycholic acid (DCA), and chenodeoxycholic acid (CDCA)] (Figure 2). $P$. bifermentans spores did not germinate in response to bile acids alone and still required ARF to activate spore germination. Interestingly, the $\mathrm{OD}$ change during $P$. bifermentans spore germination was faster in presence of $2 \mathrm{mM}$ TA (Figure 2A), similar to what was observed in C. sordellii, though the DPA release by $P$. bifermentans spores was unaffected (Figure 2B). CDCA, normally an inhibitor of germination in C. difficile, did not affect germination by $P$. bifermentans spores (Figures 2C,D). However, similar to TA, DCA increased the rate of germination measured by OD (Figure 2E) but did not influence the release of DPA (Figure 2F). To confirm that these observations are not due to a detergent-like effect of TA or DCA, spores were germinated in presence of $2 \mathrm{mM}$ Triton X-100. Triton X-100 did not increase the rate of germination by $P$. bifermentans spores either by OD (Figure 2G) or DPA release (Figure $\mathbf{2 H}$ ). These results suggest that, though TA and DCA increase the rate of OD change during germination, DPA release is unaffected by bile acids suggesting that they do not influence $P$. bifermentans spore germination and the observed effects on OD are likely an artifact.

\section{Cortex Degradation Precedes DPA Release During $P$. bifermentans Spore Germination}

A major difference between $C$. difficile and B. subtilis spore germination is the timing/order of the release of DPA and cortex fragments (Francis et al., 2015). To understand if P. bifermentans spores release cortex fragments before DPA (similar to C. difficile spore germination) or vice versa (similar to B. subtilis spore germination), we utilized an assay, that detects the presence of reducing sugars during germination, as described previously
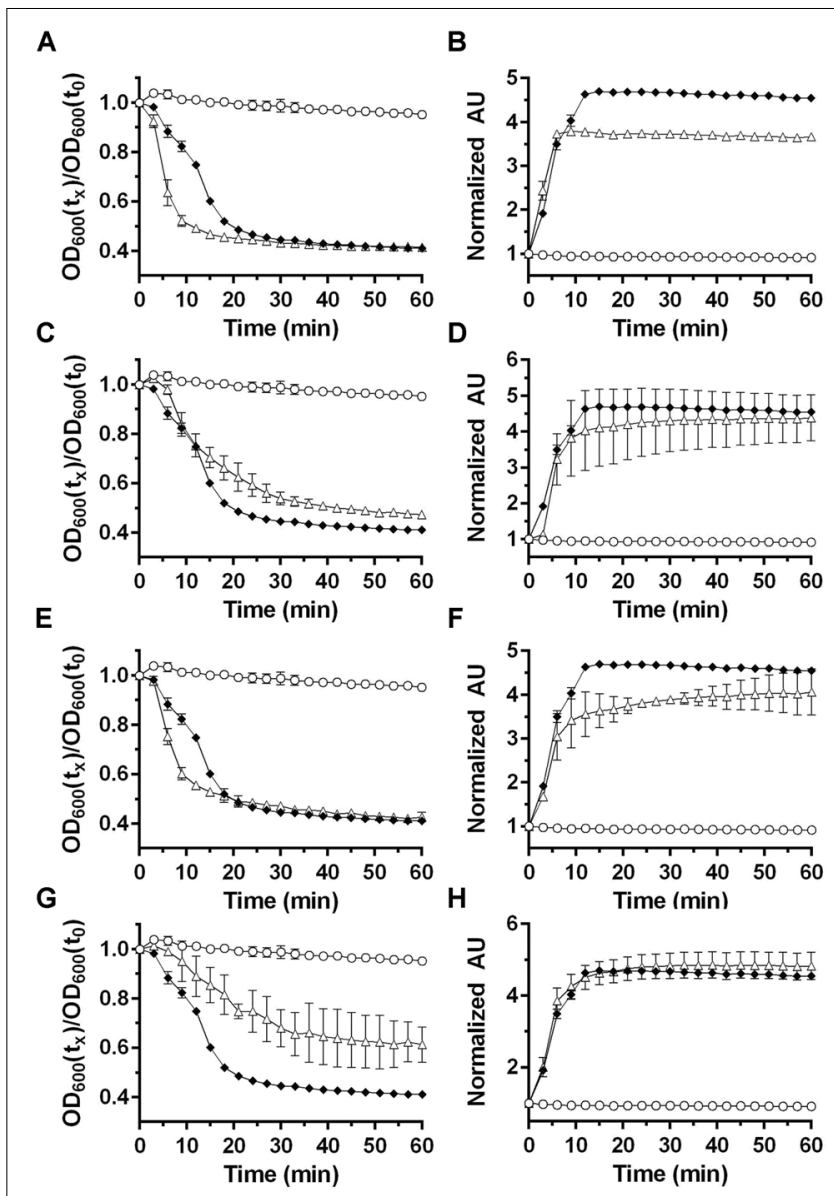

FIGURE 2 | Analyzing the influence of bile acids on P. bifermentans spore germination. Germination of $P$. bifermentans spores was analyzed by changes in $\mathrm{OD}_{600}(\mathbf{A}, \mathbf{C}, \mathbf{E}, \mathbf{G})$ and $\mathrm{Tb}^{3+}$ fluorescence upon complexing with DPA (B,D,F,H). Spores, o; $50 \mathrm{mM}$ L-alanine, $5 \mathrm{mM}$ L-phenylalanine, $5 \mathrm{mM}$ L-arginine, ; $50 \mathrm{mM} \mathrm{L-alanine,} 5 \mathrm{mM}$ L-phenylalanine, $5 \mathrm{mM}$ L-arginine, indicated compound, $\Delta$. (A,B) $2 \mathrm{mM}$ taurocholic acid; (C,D) $2 \mathrm{mM}$ chenodeoxycholic acid; (E,F) 2 mM deoxycholic acid; (G,H) 2 mM triton $X-100$. Data points represent the average from three independent experiments and error bars represent the standard deviation from the mean.

(Francis et al., 2015). Spores were suspended in germination buffer supplemented with $100 \mathrm{mM}$ ARF (the concentration of ARF was increased to $100 \mathrm{mM}$ each in order to achieve higher levels of spore germination in this assay) and germination was monitored over time. At the indicated time points, samples were removed and processed for the presence of reducing sugars and the presence of DPA. Within 2 min after the induction of germination, we observed a statistically significant difference between the amount of released reducing sugar and DPA (Figure 3). This difference was also present at $5 \mathrm{~min}$. However, by $10 \mathrm{~min}$ post-germinant addition, the fraction of released cortex fragments and DPA were indistinguishable. Because we observed the presence of reducing sugars in the germination medium before we observed the presence of DPA, these results suggest that cortex degradation occurs prior to DPA release during $P$. bifermentans spore germination. 


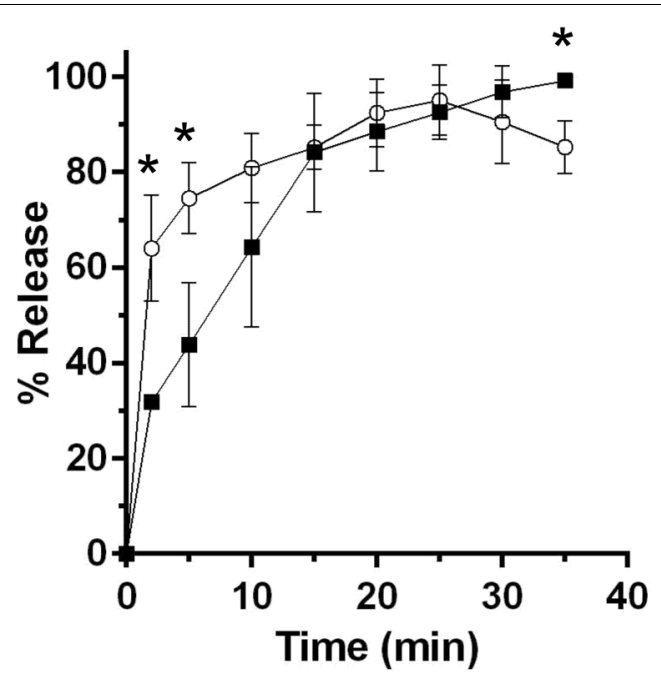

FIGURE 3 | Cortex degradation precedes DPA release during $P$. bifermentans spore germination. P. bifermentans spores were germinated in the presence of L-alanine, L-arginine, and L-phenylalanine. At the indicated times, samples were taken to analyze cortex fragment release (o) and for DPA release ( $\mathbf{\square})$. Data points represent the average from three independent experiments and error bars represent the standard deviation from the mean. Statistical significant was determined using a two-way ANOVA with Sidak's multiple comparisons test. The asterisk marked points indicate statistical significance (p-value $<0.05)$.

\section{Analyzing DPA Release in the Presence of High Concentrations of Osmolytes}

DPA release by germinating $C$. difficile spores can be delayed by high osmolyte concentrations (e.g., sorbitol) (Francis and Sorg, 2016). We hypothesized that if cortex degradation precedes DPA release during $P$. bifermentans spore germination, the release of DPA may be dependent on the osmotic changes that occur at the inner spore membrane. To test this hypothesis, we added increasing amounts of sorbitol to the germination buffer and monitored DPA release during germination by both $P$. bifermentans spores and $B$. subtilis spores (Figure 4). We could not simultaneously measure cortex degradation and DPA release during P. bifermentans spore germination. Due to unknown reasons, the ARF amino acids in the sorbitol-containing germination buffer reacted with the components of the cortex hydrolysis assay and did not yield a colorimetric signal. When P. bifermentans spores were suspended in germination buffer supplemented with $10 \%$ sorbitol (Figure 4A), we observed a short, but non-significant, delay in the release of DPA compared to spores suspended in germination buffer alone. There also was no significant delay in DPA release for germinating B. subtilis spores in $10 \%$ sorbitol (though sorbitol increased the total signal during B. subtilis spore germination; Figure 4B). When the amount of sorbitol was increased to $20 \%$ (Figure 4C), 30\% (Figure 4E), and $38 \%$ (Figure 4G), the delay in DPA release by germinating P. bifermentans spores increased by nearly 15 min (Figure 4G). For $B$. subtilis spore germination, the presence of increasing concentrations of sorbitol slightly delayed DPA release but did
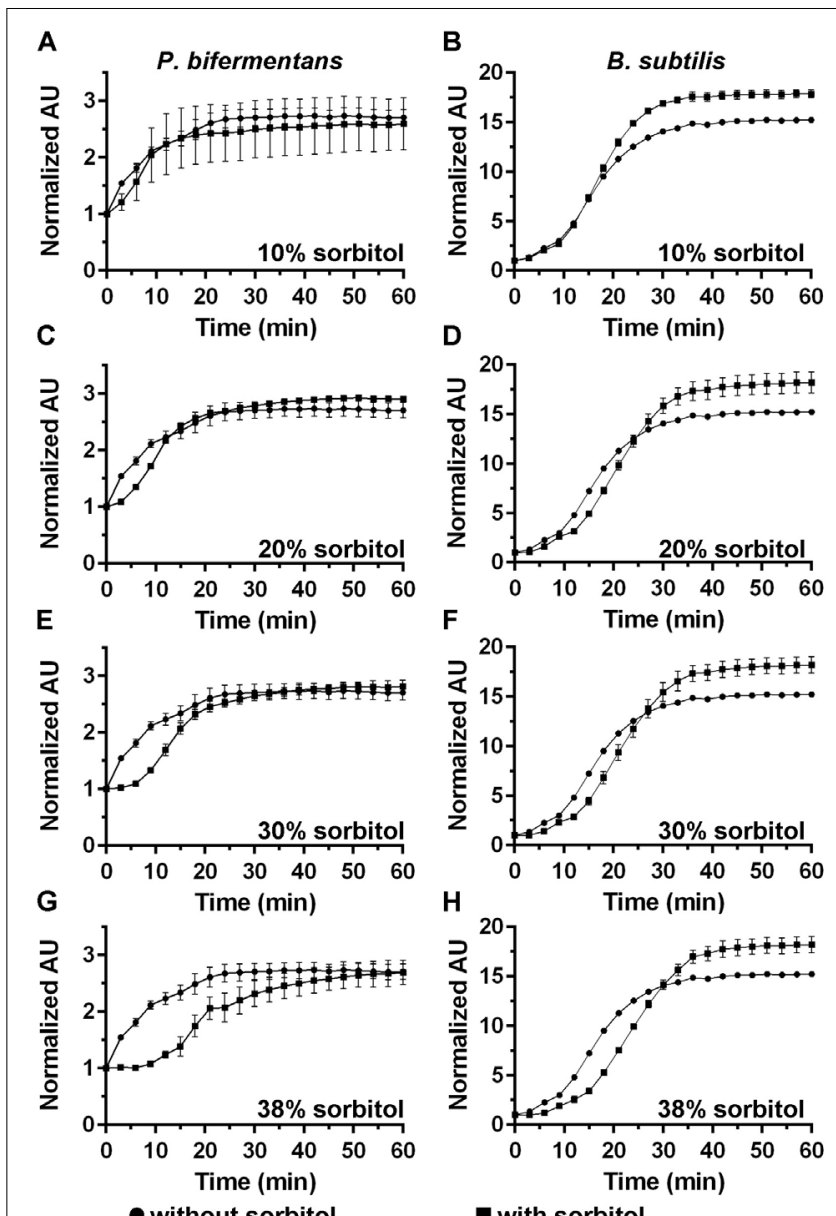

with sorbitol

FIGURE 4 | Analyzing DPA release in the presence of high concentrations of sorbitol. DPA release from germinating $P$. bifermentans spores $(\mathbf{A}, \mathbf{C}, \mathbf{E}, \mathbf{G})$ or from germinating $B$. subtilis spores $(\mathbf{B}, \mathbf{D}, \mathbf{F}, \mathbf{H})$ was analyzed germinated in presence of ( $\mathbf{\square})$ and in absence of $(\bullet)$ sorbitol. (A,B) 10\% sorbitol; (C,D) 20\% sorbitol; (E,F) 30\% sorbitol; (G,H) 38\% sorbitol. Data points represent the average from three independent experiments and error bars represent the standard deviation from the mean.

not appear to be dose-dependent as observed for P. bifermentans (Figures 4B,D,F,H).

\section{Quantifying the Effects of Sorbitol on Spore Germination}

The data in Figure 4 suggest that $P$. bifermentans spores may be more susceptible to a sorbitol-mediated delay in DPA release than B. subtilis spores. A 2-way ANOVA analysis of each curve found that a significant delay in the initial DPA release can be observed during $P$. bifermentans and B. subtilis spore germination. To quantify this effect we determined the time at which the maximum rate of DPA release occurred by taking the first order derivative of the germination plots used to derive the data in Figure 4, 5. Plotted in Figures 5A,B are the raw data from the derivative and show that $P$. bifermentans spores delay DPA release, in a dose dependent manner, upon sorbitol addition (Figure 5A). However, B. subtilis spores appear to 


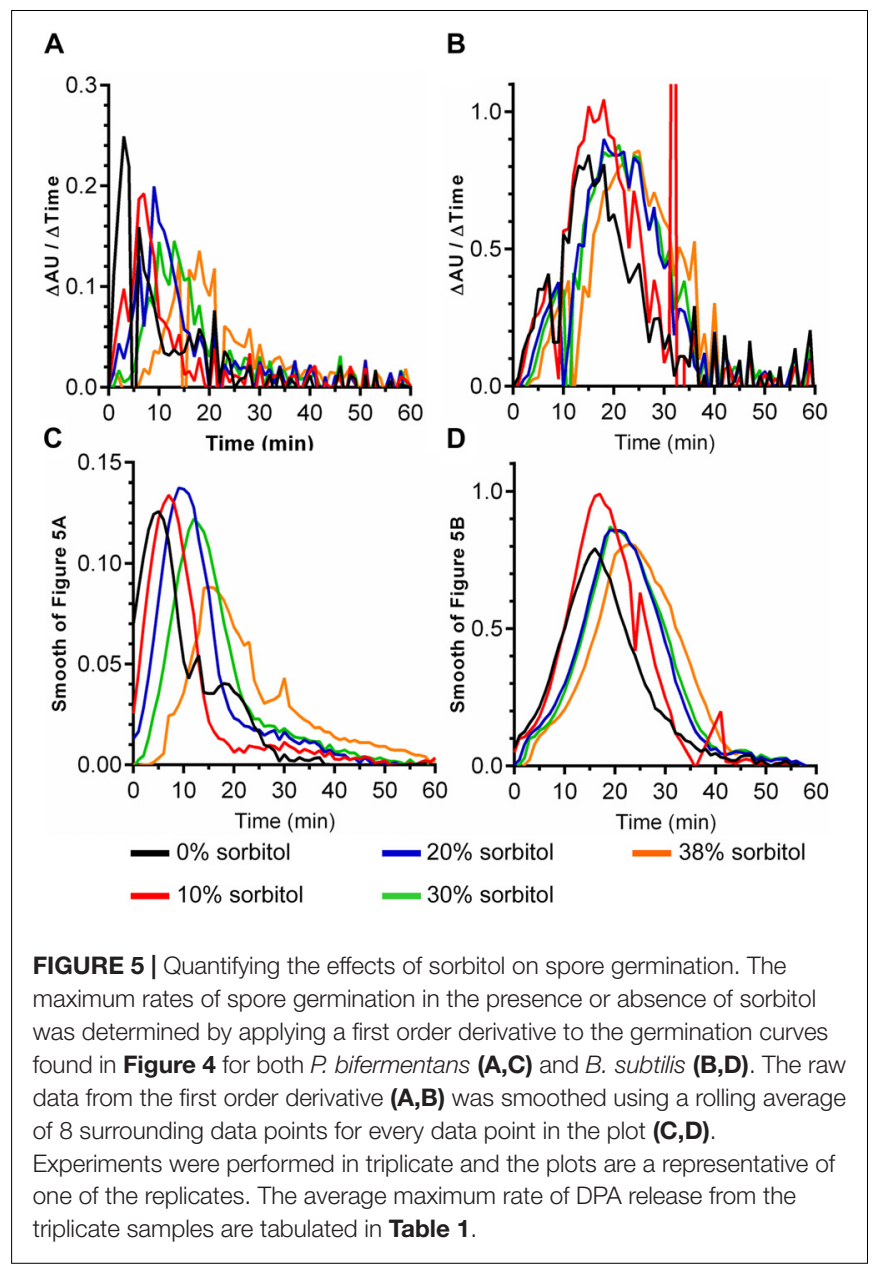

be not as influenced as $P$. bifermentans spores (Figure 5B). To provide a clearer understanding of what is occurring in Figures $\mathbf{5 A}, \mathbf{B}$, we took a rolling average of the surrounding 8 data points for every data point in $5 \mathrm{~A}$ and $5 \mathrm{~B}$ to smooth the plots (Figures 5C,D). As shown in Figure 5C and quantified in Table 1, the time at which the maximum rate of DPA release occurs during $P$. bifermentans spore germination is delayed in a step-wise manner with increasing concentrations of sorbitol. However, this same step-wise delay is not nearly as dramatic during B. subtilis spore germination (Figure 5D and Table 1). These results suggest that: (i) high osmolyte concentrations prevent the release of DPA during $P$. bifermentans spore germination similar to prior observations during $C$. difficile spore germination; (ii) that germination by $P$. bifermentans spores occurs more similar to C. difficile than B. subtilis; and (iii) that, though mechanosensing may play a role during $B$. subtilis spore germination (Velasquez et al., 2014), other factors are influencing DPA release by B. subtilis spores (e.g., germinant receptors or GerD).

\section{DISCUSSION}

Most spore-forming bacteria studied to date germinate using a mechanism similar to what has been described for B. subtilis. In $B$. subtilis, germinant recognition by the Ger-type germinant receptor results in the release of DPA from the core, likely through a channel composed of the SpoVA proteins. This event triggers the degradation of the spore cortex layer and the irreversible loss of dormancy. C. difficile spore germination is triggered by the combinatorial actions of certain bile acids and glycine (Sorg and Sonenshein, 2008; Bhattacharjee et al., 2016b). Though the hypothesized amino acid germinant receptor has not been identified, the bile acid germinant receptor is the subtilisinlike, pseudoprotease, CspC (Francis et al., 2013). Because this model differs from other models of spore germination, we hypothesized that other organisms whose csp locus is similar to that of $C$. difficile may initiate germination through this alternate pathway.

Using NCBI BLAST to search for cspBAC loci similar to C. difficile yielded a couple of Clostridial species with high genetic similarity, $P$. bifermentans and $P$. sordellii (Kevorkian et al., 2016). P. sordellii is a virulent organism which cause a range of health issues such as hemorrhagic enteritis in animals, infections due to penetrating trauma (e.g., black tar heroin use), and gynecological procedures in humans (Al-Mashat and Taylor, 1983; Clark, 2003; Kimura et al., 2004; Fischer et al., 2005; Aldape et al., 2006; Aronoff and Ballard, 2009; Ramirez and Abel-Santos, 2010). P. bifermentans, rarely, is associated with disease directly, with only 13 cases reported (Hale et al., 2016). Recently, a subspecies, P. bifermentans subsp. malaysia, was discovered and produces toxins that are active against a host of mosquito genera, especially Anopheles, carrier of malarial parasite (Charles et al., 1990). The genetic similarity between $P$. bifermentans and $P$. sordellii is high and, at one point, the two species had been classified as one organism, suggesting that their mechanisms of germination are likely shared (Brooks and Epps, 1959; Maclennan, 1962).

Similar to what is found in C. difficile, $P$. bifermentans encodes a translational fusion between $\operatorname{csp} B$ and $\operatorname{csp} A$. Encoded downstream and, likely, part of the same transcriptional unit is $\operatorname{csp} C$. Of the three encoded proteins, only CspB is

TABLE 1 | Quantifying the sorbitol-dependent delay in DPA release (min).

\begin{tabular}{lccccc}
\hline & 0\% Sorbitol & $\mathbf{1 0 \%}$ Sorbitol & $\mathbf{2 0 \%}$ Sorbitol & 30\% Sorbitol & $\mathbf{3 8 \%}$ Sorbitol \\
\hline P. bifermentans & $5 \pm 0.6$ & $7.3 \pm 0.3(2.3)$ & $9 \pm 0(4.0)$ & $12.3 \pm 0.3(7.3)$ & $17.7 \pm 2.7(12.7)$ \\
B. subtilis & $16 \pm 0$ & $16.7 \pm 0.3(0.7)$ & $20 \pm 0.6(4.0)$ & $19.7 \pm 0.7(3.7)$ & $22.3 \pm 0.7(6.3)$ \\
\hline
\end{tabular}

The time at which the maximum rate of DPA release under the conditions in Figures $5 C, D$ (smoothed data) occurred was tabulated. The values reported are the averages from three independent experiments \pm SEM. Values in parentheses highlight the delay and are tabulated by subtracting the value at 0\% sorbitol from the other values. 
predicted to have catalytic activity (Rawlings and Barrett, 1993; Rawlings et al., 2014). In CspB, the catalytic Asp, His and Ser [and the residues surrounding the triads common for Peptidase S8 family of proteases (subtilisin)] are found between amino acids 96-98, 160-163, and 458-464, respectively (Rawlings et al., 2014). For CspA, only the catalytic Asp is positioned correctly within the surrounding amino acid motif characteristic of S8 family members (amino acids 664-666 of CspBA) (Rawlings et al., 2014). Though the His and Ser residues are present, the surrounding amino acid motifs that are characteristic of the S8 family of peptidases are not in tact [the amino acid motifs surrounding the catalytic His and Ser are one amino acid and three amino acids off, respectively (Supplementary Figure S2, highlighted residues)] (Rawlings et al., 2014; Kevorkian et al., 2016). In P. bifermentans CspC, the would-be catalytic His and Ser are absent (amino acids 167-170 and 482-487, respectively) but the Asp is present (amino acids 105-108) (Rawlings et al., 2014). Thus, similar to C. difficile, only CspB has predicted catalytic activity. Though $\mathrm{CspC}$ is a pseudoprotease, CspA may have no catalytic activity or, more likely, may have reduced activity due to the motif for the triad being off by one amino acid for two of the residues.

In the NCBI database, $P$. bifermentans is predicted to encode a gerA homolog. GerA belongs to the Ger-type family of germinant receptors. Because we were interested in understanding the mechanism of $P$. bifermentans spore germination, we searched for homologs of the $P$. bifermentans GerA spore germination protein to identify other organisms with mechanisms of spore germination that might resemble $P$. bifermentans. Interestingly, though the $P$. bifermentans gene is annotated as ger $A$ in NCBI and UniProt databases, when used as a BLAST query to B. subtilis, the most closely related protein identified was not GerA, but rather, SpoVAF. Thus, it is probable that, similar to $C$. difficile, $P$. bifermentans does not encode orthologs of the ger-type germinant receptor.

Germination of $P$. bifermentans spores is initiated in response to ARF, though other combinations can stimulate spore germination (i.e., AR and AF) (Figure 1). L-alanine is the most common germinant among all, studied, spore-forming bacteria (Bhattacharjee et al., 2016b). Thus, it is not surprising that $P$. bifermentans spores initiate germination in response to $\mathrm{L}$ alanine. Waites and Wyatt (1971) previously characterized the germinants for $P$. bifermentans spores and described lactate and pyruvate as germinants. Since we observe rapid and efficient germination in response to $\mathrm{ARF}$, as measured both by $\mathrm{OD}$ change and DPA release (Figures 1C,D), we did not test pyruvate or lactate with the amino acid mixture. Because both $C$. difficile and $P$. sordellii encode csp-type receptors and both respond to steroid-based compounds (bile acids) as cues for germination, we tested if the presence of these proteins results in a spore that responds to bile acids as germinants (Sorg and Sonenshein, 2008; Liggins et al., 2011). As shown in Figure 2, the rate of $P$. bifermentans spore germination, measured by changes in $\mathrm{OD}_{600} \mathrm{~nm}$, was enhanced by both TA and DCA. However, this effect on germination was not apparent when the release of DPA was analyzed. Potentially, TA and DCA help germinants gain access to the germinant receptors or help cortex degradation. However, the reason for the impact of the bile acids on germination, measured by changes in $\mathrm{OD}_{600}$ $\mathrm{nm}$, is unknown. Thus, bile acids do not appear to impact the $P$. bifermentans spore germination and, therefore, the mere presence of the Csp proteins does not indicate that a spore germinates in response to steroid molecules. This is similar to other germinant receptors. Despite homology among the Gerfamily of germinant receptors, there are differences in the signals that stimulate germination. For example, the B. subtilis GerAA protein is $43 \%$ identical ( $62 \%$ similar) to the GerBA protein. These two germinant receptors respond, at least in part, to very different germinants (L-alanine or AGFK, respectively). The C. difficile CspBA protein is $59 \%$ identical and $75 \%$ similar to the P. bifermentans CspBA protein (Supplementary Figure S2) suggesting that, should the CspBAC proteins from $P$. bifermentans function similarly to that of $C$. difficile, these proteins could respond to different germinants (Kevorkian et al., 2016).

In prior studies, we found that the release of cortex fragments by germinating $C$. difficile spores precedes the release of DPA from the core and that high concentrations of osmolytes (i.e., sorbitol, trehalose, or sucrose) could delay the release of DPA from the core of germinating C. difficile spores (Francis et al., 2015; Francis and Sorg, 2016). Similar to what is observed during $C$. difficile spore germination, we found that cortex fragments appear in the germination solution before DPA during P. bifermentans spore germination. Moreover, the release of DPA by germinating $P$. bifermentans spores could be delayed by sorbitol in a dose-dependent manner. Because cortex degradation preceded DPA release during P. bifermentans spore germination, these results suggest that DPA release by germinating $P$. bifermentans spores occurs in a mechanosensing fashion. Because the rate of DPA release B. subtilis was not as affected by high concentrations of osmolyte, mechanosensing is likely not important for DPA release during nutrient-mediated germination; DPA-mediated germination likely would stimulate cortex degradation and thus release of DPA from the core in a mechanosensing fashion (Setlow, 2013; Velasquez et al., 2014).

Prior work done by Kevorkian et al. (2016) revealed that the Peptostreptococcaceae family members conserve a catalytically dead CspC protein but the CspBA proteins vary in their hypothesized protease activities. For example, $C$. difficile encodes a functional $\mathrm{CspB}$ protein $(\mathrm{F})$ fused to a non-functional CspA protein $(\mathrm{N})$. This $\mathrm{FN}$ arrangement is conserved across all C. difficile isolates, but is not universally conserved in all Peptostreptococcaceae. For P. bifermentans, the authors found an arrangement of a non-functional $\mathrm{CspB}$ but a potentially functional CspA. Based upon our findings here, CspB is likely to be catalytically active (due to the sequencing error in the published genome) and CspA may be inactive or exhibit reduced activity (see above). But, pseudoprotease regulation of spore germination may be a common feature of the Peptostreptococcaceae family. Moreover, in the absence of Ger-family germinant receptors, organisms that encode Csp pseudoproteases may germinate in an "outside-in" mechanism. 
In summary, we found that $P$. bifermentans spore germination occurs most similarly to that observed in C. difficile. Unfortunately, due to the lack of a genetic system in $P$. bifermentans, we could not directly test the effects of mutations introduced into the $\operatorname{csp} B A$ or $\operatorname{csp} C$ coding regions. However, based on the appearance of cortex fragments before DPA during germination of $P$. bifermentans spores and that high osmolyte concentrations can delay the release of DPA, we predict that $P$. bifermentans spore germination proceeds through the same pathway as observed during $C$. difficile spore germination. These findings build upon the hypothesis that Csp-type germinant receptor activation stimulates spore germination though an 'outside-in' direction and represents a novel germination pathway involving pseudoproteases.

\section{MATERIALS AND METHODS}

\section{Bacterial Strains}

Wild type P. bifermentans ATCC 19299 was purchased from the American Type Culture Collection (ATCC) and grown in an anaerobic atmosphere $\left(10 \% \mathrm{H}_{2}, 5 \% \mathrm{CO}_{2}\right.$, and $\left.85 \% \mathrm{~N}_{2}\right)$ at $37^{\circ} \mathrm{C}$ on Difco Reinforced Clostridial Medium agar (RCM) medium, as recommended by ATCC. B. subtilis PS533 was grown on Difco sporulation medium (DSM) and LB medium.

\section{Sporulation}

$P$. bifermentans cells were streaked onto pre-reduced DuncanStrong Sporulation Media (DSSM) agar plates (Duncan and Strong, 1968) under anaerobic conditions at $37^{\circ} \mathrm{C}$. The cells were allowed to grow for 3-4 days before harvesting by scraping the growth into sterile water. B. subtilis cells were streaked onto DSM agar medium and allowed to grow for 4 days at $30^{\circ} \mathrm{C}$, as described previously (Francis et al., 2015), and harvested as described above.

\section{Spore Purification}

Spores, vegetative cells, debris, and any agar that contaminated the harvested preparation ( $P$. bifermentans and B. subtilis grew into the agar surface) were stored overnight at $4^{\circ} \mathrm{C}$. Agar was removed from the scraped spores by incubating the suspension at $75^{\circ} \mathrm{C}$ for $1 \mathrm{~h}$, as described previously (Francis et al., 2015). The resulting suspension was washed 5 times in sterile water and purified on $60 \%(\mathrm{w} / \mathrm{v})$ sucrose solution as described previously (Bhattacharjee et al., 2016a). Purified spores were again washed 5 times and stored in $1 \mathrm{~mL}$ sterile water. Purified spores appeared phase bright and did not contain observable vegetative cells.

\section{Spore Germination}

Purified $P$. bifermentans spores were heated for $30 \mathrm{~min}$ at $75^{\circ} \mathrm{C}$ (heat activation was required for $P$. bifermentans spore germination; Supplementary Figure S3) and B. subtilis spores at $80^{\circ} \mathrm{C}$ prior to germination (Keynan et al., 1964; Francis et al., 2015). Spores were added to Falcon clear 96 well plates containing germination buffer (50 mM HEPES pH 7.5, $100 \mathrm{mM} \mathrm{NaCl}$ ) alone or in buffer supplemented with germinants $(50 \mathrm{mM} \mathrm{L}$-alanine, $5 \mathrm{mM} \mathrm{L}$-phenylalanine, $5 \mathrm{mM}$ L-arginine for $P$. bifermentans;
$100 \mathrm{mM}$ L-valine for B. subtilis). Sorbitol was added where indicated. Germination of the $\mathrm{OD}_{600}=0.5-0.7$ spore suspension was measured over time at $\mathrm{OD}_{600} \mathrm{~nm}$ in SpectraMax M3 plate reader at $37^{\circ} \mathrm{C}$.

The release of DPA from $\mathrm{OD}_{600}=0.25-0.3$ spores was measured by adding $250 \mu \mathrm{M}$ terbium chloride (final concentration) to germination buffer (above) with or without added germinants and/or osmolyte, as described previously (Bhattacharjee et al., 2016a). Terbium $\left(\mathrm{Tb}^{3+}\right.$ ) fluorescence was monitored using a SpectraMax M3 plate reader with excitation at $270 \mathrm{~nm}$ and emission at $545 \mathrm{~nm}$ with a $420 \mathrm{~nm}$ cut-off as previously described (Bhattacharjee et al., 2016a).

\section{Cortex Hydrolysis}

Cortex hydrolysis assay was performed for $P$. bifermentans spores, as previously published (Francis et al., 2015). Spores were suspended in germination buffer at $\mathrm{OD}_{600} \sim 3$. However, the germinant concentrations were increased to $100 \mathrm{mM}$ each of Lalanine, L-phenylalanine, and L-arginine to increase germination of the spores.

\section{Statistical Analyses}

Data points represent the average from three, independent experiments and error bars represent the standard deviation from the mean. Statistical significance was determined using a two-way ANOVA with Sidak's multiple comparisons test.

\section{AUTHOR CONTRIBUTIONS}

DB performed the experiments. DB and JS analyzed the data and wrote the manuscript.

\section{FUNDING}

This project was supported by awards 5R01AI116895 and 1U01AI124290 to JS from the National Institute of Allergy and Infectious Diseases. The content is solely the responsibility of the authors and does not necessarily represent the official views of the NIAID. The funders had no role in study design, data collection and interpretation, or the decision to submit the work for publication.

\section{ACKNOWLEDGMENTS}

The authors would like to thank members of the Sorg and Dr. Leif Smith laboratories at Texas A\&M University for their helpful comments during the preparation of this manuscript. The authors thank Dr. Michael Francis for technical advice.

\section{SUPPLEMENTARY MATERIAL}

The Supplementary Material for this article can be found online at: https://www.frontiersin.org/articles/10.3389/fmicb.2018. 02487/full\#supplementary-material 


\section{REFERENCES}

Adams, C. M., Eckenroth, B. E., Putnam, E. E., Doublie, S., and Shen, A. (2013). Structural and functional analysis of the $\mathrm{CspB}$ protease required for Clostridium spore germination. PLoS Pathog. 9:e1003165. doi: 10.1371/journal.ppat.1003165 doi: 10.1371/journal.ppat.1003165

Aldape, M. J., Bryant, A. E., and Stevens, D. L. (2006). Clostridium sordellii infection: epidemiology, clinical findings, and current perspectives on diagnosis and treatment. Clin. Infect. Dis. 43, 1436-1446. doi: 10.1086/508866

Al-Mashat, R. R., and Taylor, D. J. (1983). Production od diarrhoea and enteric lesions in calves by the oral inoculation of pure cultures of Clostridium sordellii. Vet. Rec. 112, 141-146. doi: 10.1136/vr.112.7.141

Aronoff, D. M., and Ballard, J. D. (2009). Clostridium sordellii toxic shock syndrome. Lancet Infect. Dis. 9, 725-726. doi: 10.1016/S1473-3099(09)70303-2

Bhattacharjee, D., Francis, M. B., Ding, X., McAllister, K. N., Shrestha, R., and Sorg, J. A. (2016a). Reexamining the germination phenotypes of several Clostridium difficile strains suggests another role for the CspC germinant receptor. J. Bacteriol. 198, 777-786. doi: 10.1128/JB.00908-15

Bhattacharjee, D., McAllister, K. N., and Sorg, J. A. (2016b). Germinants and their receptors in Clostridia. J. Bacteriol. 198, 2767-2775. doi: 10.1128/JB.00405-16

Brooks, M. E., and Epps, H. B. (1959). Taxonomic studies of the genus Clostridium: Clostrididum bifermentans and C. sordellii. J. Gen. Microbiol. 21, 144-155. doi: 10.1099/00221287-21-1-144

Charles, J. F., Nicolas, L., Sebald, M., and de Barjac, H. (1990). Clostridium bifermentans serovar malaysia: sporulation, biogenesis of inclusion bodies and larvicidal effect on mosquito. Res. Microbiol. 141, 721-733. doi: 10.1016/09232508(90)90066-Y

Clark, S. (2003). Sudden death in periparturient sheep associated with Clostridium sordellii. Vet. Rec. 153:340.

Duncan, C. L., and Strong, D. H. (1968). Improved medium for sporulation of Clostridium perfringens. Appl. Microbiol. 16, 82-89.

Errington, J. (2003). Regulation of endospore formation in Bacillus subtilis. Nat. Rev. Microbiol. 1, 117-126. doi: 10.1038/nrmicro750

Fimlaid, K. A., Bond, J. P., Schutz, K. C., Putnam, E. E, Leung, J. M., Lawley, T. D., et al. (2013). Global analysis of the sporulation pathway of Clostridium difficile. PLoS Genet. 9:e1003660. doi: 10.1371/journal.pgen.1003660

Fischer, M., Bhatnagar, J., Guarner, J., Reagan, S., Hacker, J. K., Van Meter, S. H., et al. (2005). Fatal toxic shock syndrome associated with Clostridium sordellii after medical abortion. N. Engl. J. Med. 353, 2352-2360. doi: 10.1056/ NEJMoa051620

Francis, M. B., Allen, C. A., Shrestha, R., and Sorg, J. A. (2013). Bile acid recognition by the Clostridium difficile germinant receptor, $\mathrm{CspC}$, is important for establishing infection. PLoS Pathog. 9:e1003356. doi: 10.1371/journal.ppat. 1003356

Francis, M. B., Allen, C. A., and Sorg, J. A. (2015). Spore cortex hydrolysis precedes dipicolinic acid release during Clostridium difficile spore germination. J. Bacteriol. 197, 2276-2283. doi: 10.1128/JB.02575-14

Francis, M. B., and Sorg, J. A. (2016). Dipicolinic acid release by germinating Clostridium difficile spores occurs through a mechanosensing mechanism. mSphere 1:e00306-16. doi: 10.1128/mSphere.00306-16

Gibbs, P. A. (1964). Factors affecting the germination of spores of Clostridium bifermentans. J. Gen. Microbiol. 37, 41-48. doi: 10.1099/00221287-37-1-41

Hale, A., Kirby, J. E., and Albrecht, M. (2016). Fatal spontaneous Clostridium bifermentans necrotizing endometritis: a case report and literature review of the pathogen. Open Forum Infect. Dis. 3:ofw095. doi: 10.1093/ofid/ofw095

Howerton, A., Ramirez, N., and Abel-Santos, E. (2011). Mapping interactions between germinants and Clostridium difficile spores. J. Bacteriol. 193, 274-282. doi: 10.1128/JB.00980- 10

Kevorkian, Y., Shirley, D. J., and Shen, A. (2016). Regulation of Clostridium difficile spore germination by the CspA pseudoprotease domain. Biochimie 122, 243-254. doi: 10.1016/j.biochi.2015.07.023

Keynan, A., Evanchik, Z., Halvorson, H. O., and Hastings, J. W. (1964). Activation of bacterial endospores. J. Bacteriol. 88, 313-318.

Kimura, A. C., Higa, J. I., Levin, R. M., Simpson, G., Vargas, Y., and Vugia, D. J. (2004). Outbreak of necrotizing fasciitis due to Clostridium sordellii among black-tar heroin users. Clin. Infect. Dis. 38, e87-e91. doi: 10.1086/383471

Kochan, T. J., Somers, M. J., Kaiser, A. M., Shoshiev, M. S., Hagan, A. K., Hastie, J. L., et al. (2017). Intestinal calcium and bile salts facilitate germination of
Clostridium difficile spores. PLoS Pathog. 13:e1006443. doi: 10.1371/journal. ppat.1006443

Lawson, P. A., Citron, D. M., Tyrrell, K. L., and Finegold, S. M. (2016). Reclassification of Clostridium difficile as Clostridioides difficile (Hall and O’Toole 1935) Prevot 1938. Anaerobe 40, 95-99. doi: 10.1016/j.anaerobe.2016. 06.008

Liggins, M., Ramirez, N., Magnuson, N., and Abel-Santos, E. (2011). Progesterone analogs influence germination of Clostridium sordellii and Clostridium difficile spores in vitro. J. Bacteriol. 193, 2776-2783. doi: 10.1128/JB.00058-11

Maclennan, J. D. (1962). The histotoxic clostridial infections of man. Bacteriol. Rev. 26, 177-276.

Masayama, A., Hamasaki, K., Urakami, K., Shimamoto, S., Kato, S., Makino, S., et al. (2006). Expression of germination-related enzymes, CspA, CspB, CspC, SleC, and SleM, of Clostridium perfringens S40 in the mother cell compartment of sporulating cells. Genes Genet. Syst. 81, 227-234. doi: 10.1266/ggs. 81.227

Miyata, S., Kozuka, S., Yasuda, Y., Chen, Y., Moriyama, R., Tochikubo, K., et al. (1997). Localization of germination-specific spore-lytic enzymes in Clostridium perfringens $\mathrm{S} 40$ spores detected by immunoelectron microscopy. FEMS Microbiol. Lett. 152, 243-247. doi: 10.1111/j.1574-6968.1997.tb10434.x

Moir, A., Corfe, B. M., and Behravan, J. (2002). Spore germination. Cell. Mol. Life Sci. 59, 403-409. doi: 10.1007/s00018-002-8432-8

Oren, A., and Garrity, G. M. (2016). Notification that new names of prokaryotes, new combinations, and new taxonomic opinions have appeared in volume 66, part 9, of the IJSEM. Int. J. Syst. Evol. Microbiol. 66, 4921-4923. doi: 10.1099/ ijsem.0.001620

Paredes-Sabja, D., Setlow, P., and Sarker, M. R. 2011. Germination of spores of Bacillales and Clostridiales species: mechanisms and proteins involved. Trends Microbiol. 19, 85-94. doi: 10.1016/j.tim.2010.10.004

Paredes-Sabja, D, Setlow P, Sarker MR. (2009). The protease CspB is essential for initiation of cortex hydrolysis and dipicolinic acid (DPA) release during germination of spores of Clostridium perfringens type A food poisoning isolates. Microbiology 155, 3464-3472. doi: 10.1099/mic.0.030965-0

Paredes-Sabja, D., Shen, A., and Sorg, J. A. (2014). Clostridium difficile spore biology: sporulation, germination, and spore structural proteins. Trends Microbiol. 22, 406-416. doi: 10.1016/j.tim.2014.04.003

Permpoonpattana, P., Tolls, E. H., Nadem, R., Tan, S., Brisson, A., and Cutting, S. M. (2011). Surface layers of Clostridium difficile endospores. J. Bacteriol. 193, 6461-6470. doi: 10.1128/JB.05182-11

Qureshi, N., Chawla, S., Likitvivatanavong, S., and Lee, H. L., Gill, S. S. (2014). The cry toxin operon of Clostridium bifermentans subsp. malaysia is highly toxic to Aedes larval mosquitoes. Appl. Environ. Microbiol. 80, 5689-5697. doi: 10.1128/AEM.01139-14

Ramirez, N., and Abel-Santos, E. (2010). Requirements for germination of Clostridium sordellii spores in vitro. J. Bacteriol. 192, 418-425. doi: 10.1128/JB. 01226-09

Rawlings, N. D., and Barrett, A. J. (1993). Evolutionary families of peptidases Biochem. J. 290(Pt 1):205-218. doi: 10.1042/bj2900205

Rawlings, N. D., Waller, M., Barrett, A. J., and Bateman, A. (2014). MEROPS: the database of proteolytic enzymes, their substrates and inhibitors. Nucleic Acids Res. 42, D503-D509. doi: 10.1093/nar/gkt953

Sebaihia, M., Wren, B. W., Mullany, P., Fairweather, N. F., Minton, N., Stabler, R., et al. (2006). The multidrug-resistant human pathogen Clostridium difficile has a highly mobile, mosaic genome. Nat. Genet. 38, 779-786. doi: 10.1038/ng1830

Setlow, P. (2013). Summer meeting 201-when the sleepers wake: the germination of spores of Bacillus species. J. Appl. Microbiol. 115, 1251-1268. doi: 10.1111/ jam. 12343

Setlow, P. (2014). Germination of spores of Bacillus species: what we know and do not know. J. Bacteriol. 196, 1297-1305. doi: 10.1128/JB.01455-13

Shimamoto, S., Moriyama, R., Sugimoto, K., Miyata, S., and Makino, S. (2001). Partial characterization of an enzyme fraction with protease activity which converts the spore peptidoglycan hydrolase (SleC) precursor to an active enzyme during germination of Clostridium perfringens $\mathrm{S} 40$ spores and analysis of a gene cluster involved in the activity. J. Bacteriol. 183, 3742-3751. doi: 10.1128/JB.183.12.3742-3751.2001

Shrestha, R., Lockless, S. W., and Sorg, J. A. (2017). A Clostridium difficile alanine racemase affects spore germination and accommodates serine as a substrate. J. Biol. Chem. 292, 10735-10742. doi: 10.1074/jbc.M117.791749 
Shrestha, R., and Sorg, J. A. (2018). Hierarchical recognition of amino acid co-germinants during Clostridioides difficile spore germination. Anaerobe 49, 41-47. doi: 10.1016/j.anaerobe.2017.12.001

Sorg, J. A., and Sonenshein, A. L. (2008). Bile salts and glycine as cogerminants for Clostridium difficile spores. J. Bacteriol. 190, 2505-2512. doi: 10.1128/JB. 01765-07

Sorg, J. A., and Sonenshein, A. L. (2009). Chenodeoxycholate is an inhibitor of Clostridium difficile spore germination. J. Bacteriol. 191, 1115-1117. doi: 10. 1128/JB.01260-08

Sorg, J. A., and Sonenshein, A. L. (2010). Inhibiting the initiation of Clostridium difficile spore germination using analogs of chenodeoxycholic acid, a bile acid. J. Bacteriol. 192, 4983-4990. doi: 10.1128/JB.00610-10

Velasquez, J., Schuurman-Wolters, G., Birkner, J. P., Abee, T., and Poolman, B. (2014). Bacillus subtilis spore protein SpoVAC functions as a mechanosensitive channel. Mol. Microbiol. 92, 813-823. doi: 10.1111/mmi.12591
Waites, W. M., and Wyatt, L. R. (1971). Germination of spores of Clostridium bifermentans by certain amino acids, lactate and pyruvate in the presence of sodium or potassium ions. J. Gen. Microbiol. 67, 215-222. doi: 10.1099/ 00221287-67-2-215

Conflict of Interest Statement: The authors declare that the research was conducted in the absence of any commercial or financial relationships that could be construed as a potential conflict of interest.

Copyright $\odot 2018$ Bhattacharjee and Sorg. This is an open-access article distributed under the terms of the Creative Commons Attribution License (CC BY). The use, distribution or reproduction in other forums is permitted, provided the original author(s) and the copyright owner(s) are credited and that the original publication in this journal is cited, in accordance with accepted academic practice. No use, distribution or reproduction is permitted which does not comply with these terms. 